UT-03-21

hep-th/0306096

June, 2003

\title{
Toward Open-Closed String Theoretical Description of Rolling Tachyon
}

\author{
Kazuki Ohmori ${ }^{1}$ \\ Department of Physics, Faculty of Science, University of Tokyo \\ Hongo 7-3-1, Bunkyo-ku, Tokyo 113-0033, Japan
}

\begin{abstract}
We consider how the time-dependent decay process of an unstable D-brane should be described in the full (quantum) open-closed string theory. It is argued that the system, starting from the unstable D-brane configuration, will evolve in time into the time-independent open string tachyon vacuum configuration which we assume to be finite, with the total energy conserved. As a concrete realization of this idea, we construct a toy model describing the open and closed string tachyons which admits such a time-dependent solution. The structure of our model has some resemblance to that of open-closed string field theory.
\end{abstract}

\footnotetext{
${ }^{1}$ E-mail: ohmori@hep-th.phys.s.u-tokyo.ac.jp
} 


\section{Introduction}

In a series of papers [1, 2] Sen has proposed ways to describe the classical time evolution of the open string tachyon field $T(t)$ on an unstable D-brane, at strictly zero string coupling $g_{s}=0$. Some of the results obtained using the BCFT method [1] were also reproduced from an effective field theory [2] and boundary string field theory [3]. Since the closed string degrees of freedom, which are responsible for the dissipation of the energy liberated from the D-brane, are not taken into account there, the energy must be stored in the open string sector in spite of the fact that the D-brane is decaying. In order for the energy to be conserved in the settings of [2, 3], it turns out that the 'velocity' of the tachyon field must approach a finite value $\dot{T}(t) \rightarrow 1$ in the asymptotic future $t \rightarrow \infty$. This is possible only when the tachyon potential has its minimum at infinity. Then, what happens in theories where the minimum of the tachyon potential is at a finite point in the field configuration space, such as open string field theory and $p$-adic string theory? The authors who have tried to seek rolling solutions in such theories have found the oscillations with ever-growing amplitude, typically of the form $T(t)=\sum_{n} a_{n} e^{n t}$ [4, [5] [. It is possible that these seemingly different behaviors of the tachyon field are related to each other through some field redefinition, but at present we do not know precisely how to reconcile them. Furthermore, it has been suggested that, once the non-zero string coupling $g_{s}$ is turned on, the energy which was initially stored in the open string sector is converted to the closed string radiation, no matter how small $g_{s}$ is [7, 8].

In this paper, we will try to attack the problem of rolling tachyon from the viewpoint of open-closed string theory. For simplicity we will restrict ourselves to the spatially homogeneous decay of an unstable space-filling D-brane. And we assume that the tachyon vacuum is at a finite point in the field configuration space, in which case much more definite discussion is possible than in the case of the runaway potential. Then, we expect the following properties for the open string tachyon condensation in open-closed string theory:

1. There are two homogeneous and static (i.e. constant in spacetime) solutions corresponding to the unstable D-brane background and the open string tachyon vacuum where the D-brane has disappeared. These two configurations should have the same energy density because the energy initially stored in the D-brane (=the open string modes) is just transferred to the closed string modes.

2. The rolling of the tachyon is described by a time-dependent solution which interpolates between the above two vacuum configurations. In particular, since the open string sector can lose its energy, it would be natural to consider that the tachyon field will eventually relax in the (finite) tachyon vacuum.

As is clear from the appearance of a factor of $\sqrt{\hbar}$ in the open-closed string action (3), we must 
go beyond the classical level to incorporate the interactions between open and closed strings. ${ }^{2}$ Since it is quite difficult to deal with the full open-closed string field theory (see [10, 11] and references therein), here we consider a simple model including tachyons only, as a first step toward the ultimate goal. In the next section we introduce the model and show that it admits a rolling solution satisfying the properties mentioned above. Section 3 is devoted to summary and discussion.

\section{A Toy Model for Open and Closed String Tachyons}

Let us consider a model described by the following action, ${ }^{3}$

$$
\begin{aligned}
S & =\int d^{D} x \mathcal{L}=\int d^{D} x\left(\mathcal{L}_{o}+\mathcal{L}_{c}+\mathcal{L}_{\text {int }}\right) \\
\mathcal{L}_{o} & =\frac{1}{2} \phi \square \phi+\frac{1}{2} \phi^{2} \\
\mathcal{L}_{c} & =\frac{1}{2} \psi \square \psi+2 \psi^{2} \\
\mathcal{L}_{\text {int }} & =-\frac{1}{3} \widetilde{\phi}^{3}+c_{2} \widetilde{\phi} \widetilde{\psi}-\widetilde{\phi}^{2} \widetilde{\psi}
\end{aligned}
$$

where $\phi$ and $\psi$ are the open and closed string tachyon fields respectively, and we have defined

$$
\widetilde{A}(x) \equiv e^{(\log K) \square} A(x)=K^{\square} A(x),
$$

with $K=2$. We take the flat spacetime metric to be $\eta^{\mu \nu}=\operatorname{diag}(-1,1, \cdots, 1)$, so that the d'Alembertian is $\square=-\partial_{t}^{2}+\nabla^{2}$. We do not need to specify the spacetime dimensionality $D$. $c_{2}$ is a constant which will be fixed below.

One may notice that the structure of the action (11), in particular the ${ }^{\sim}$-operation (2), is reminiscent of the open-closed string field theory action [10]

$$
\begin{aligned}
S_{\mathrm{OCSFT}} & \sim\left(\left\langle\Phi\left|Q_{B}\right| \Phi\right\rangle+g_{o}\left\langle\Phi^{3}\right\rangle_{\text {disk }}+g_{o}^{2}\left\langle\Phi^{4}\right\rangle_{\text {disk }}\right)+\left\langle\Psi\left|\left(c_{0}-\bar{c}_{0}\right)\left(Q_{B}+\bar{Q}_{B}\right)\right| \Psi\right\rangle \\
& +\sqrt{\hbar}\left(g_{c}\left\langle\Psi^{3}\right\rangle_{\text {sphere }}+\langle\Psi\rangle_{\text {disk }}+g_{o}\langle\Phi \Psi\rangle_{\text {disk }}+g_{o}^{2}\left\langle\Phi^{2} \Psi\right\rangle_{\text {disk }}\right)+\mathcal{O}\left(g_{o}^{3}, \hbar\right)
\end{aligned}
$$

where $\Phi$ and $\Psi$ are open and closed string fields, respectively. By truncating the string fields as

$$
|\Phi\rangle=\int \frac{d^{D} k}{(2 \pi)^{D}} \phi(k) c_{1}|k\rangle, \quad|\Psi\rangle=\int \frac{d^{D} k}{(2 \pi)^{D}} \psi(k) c_{1} \bar{c}_{1}|k\rangle,
$$

and substituting them into the action (3), we would obtain the lagrangian whose structure is similar to (11). ${ }^{4}$ However, there should be some discrepancies between the resulting action and

\footnotetext{
${ }^{2}$ For a recent discussion about the quantum aspects of Witten's cubic open string field theory, see 9 .

${ }^{3}$ We are working in units where $\alpha^{\prime}=1$.

${ }^{4}$ We have approximately calculated the open-closed transition vertex with the stub and the strip omitted, and found that it is roughly estimated as $\langle\Phi \Psi\rangle_{\text {disk }} \sim \check{\phi}(x) \check{\psi}(x)$ with $\check{A}(x) \simeq \exp ((\log 1.352) \square) A(x)$. It is quite difficult to compute the open-open-closed vertex $\left\langle\Phi^{2} \Psi\right\rangle_{\text {disk }}$ because it includes the integration over the moduli space.
} 
(11). In particular, there is no reason why $\psi, \psi^{3}$ and $\phi^{4}$ terms can be neglected. Therefore we consider the action (11) as just a toy model, instead of having been derived from the open-closed string field theory action via level truncation.

The vacuum structure of this model can be studied by looking at the potential

$$
\mathcal{V}=-\left.\mathcal{L}\right|_{\phi, \psi=\text { const. }}=-\frac{1}{2} \phi^{2}-2 \psi^{2}+\frac{1}{3} \phi^{3}-c_{2} \phi \psi+\phi^{2} \psi
$$

If we eliminate the closed string tachyon field by its equation of motion $\psi=\frac{1}{4}\left(\phi^{2}-c_{2} \phi\right)$, we get the effective potential for $\phi$,

$$
\mathcal{V}_{\text {eff }}=\frac{1}{8} \phi^{2}\left\{\left(\phi+\frac{4}{3}-c_{2}\right)^{2}+\frac{8}{3} c_{2}-\frac{52}{9}\right\} .
$$

From this expression, we find that, if we choose $c_{2}=\frac{13}{6}$, the effective potential takes the form $\mathcal{V}_{\text {eff }}=\frac{\phi^{2}}{8}\left(\phi-\frac{5}{6}\right)^{2}$, so that $\mathcal{V}_{\text {eff }}$ has two degenerate vacua at $\phi=0$ and $\frac{5}{6}$. With this value of $c_{2}$, the potential (5) has three stationary points,

$$
\begin{array}{rlll}
\text { solution }(\mathrm{I}) & : & \left(\phi^{\mathrm{I}}, \psi^{\mathrm{I}}\right)=(0,0), & \mathcal{V}=0 \\
\text { solution }(\mathrm{II}) & : & \left(\phi^{\mathrm{II}}, \psi^{\mathrm{II}}\right)=\left(\frac{5}{6},-\frac{5}{18}\right), & \mathcal{V}=0 \\
\text { solution }(\mathrm{III}) & : & \left(\phi^{\mathrm{III}}, \psi^{\mathrm{III}}\right)=\left(\frac{5}{12},-\frac{35}{192}\right), & \mathcal{V}=\frac{625}{165888}
\end{array}
$$

where the value of $\mathcal{V}$ for each solution shows the height of the potential there.

Next we will explain that the solutions (I) and (II) can be regarded as representing the Dbrane and the open string tachyon vacuum, respectively. First, note that there is a $\phi-\psi$ mixing term in the action (11) at the quadratic level. In order to determine the perturbative spectrum around any one of the solutions, we must diagonalize it. Let us start with the solution (I). After the Fourier transformation, the quadratic part of the action (11) can be arranged as

$$
\begin{aligned}
& S_{\text {quad }}^{\mathrm{I}}=-\frac{1}{2} \int \frac{d^{D} k}{(2 \pi)^{D}}(\phi(-k), \psi(-k)) \mathcal{M}^{\mathrm{I}}\left(k^{2}\right)\left(\begin{array}{c}
\phi(k) \\
\psi(k)
\end{array}\right), \\
& \mathcal{M}^{\mathrm{I}}\left(k^{2}\right)=\left(\begin{array}{cc}
k^{2}-1 & -\frac{13}{6} K^{-2 k^{2}} \\
-\frac{13}{6} K^{-2 k^{2}} & k^{2}-4
\end{array}\right) .
\end{aligned}
$$

The mass spectrum is found by looking for the values of $k^{2}=-m^{2}$ at which the eigenvalues of the matrix $\mathcal{M}^{\mathrm{I}}\left(k^{2}\right)$ vanish. This can be equivalently accomplished by solving $\operatorname{det} \mathcal{M}^{\mathrm{I}}\left(k^{2}\right)=0$. Since we cannot solve this equation analytically, we resort to the numerical study. We see from Fig. 1, where $\operatorname{det} \mathcal{M}^{\mathrm{I}}$ is shown as a function of $k^{2}$, that there is a closed string tachyon state with $m_{c}^{2} \simeq-4.000$, and an open string tachyon state with $m_{o}^{2} \simeq-0.863$. We therefore consider the solution (I) as the unstable D-brane background. We do not concern ourselves about an extra state around $m^{2}=-0.110$ which is not important for our purpose.

Now we turn to the solution (II). When we expand the fields as

$$
\phi=\phi^{\mathrm{II}}+\phi^{\prime}, \quad \psi=\psi^{\mathrm{II}}+\psi^{\prime},
$$




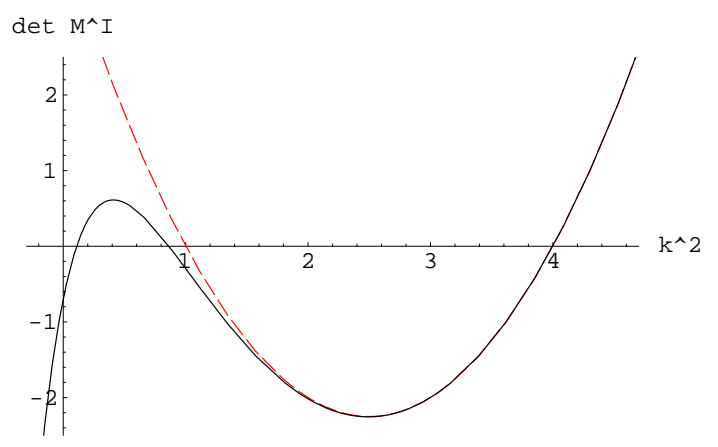

Figure 1: $\operatorname{det} \mathcal{M}^{\mathrm{I}}$ is plotted as a function of $k^{2}$ (solid line). If the $\phi-\psi$ mixing term were absent, the determinant would behave like the dashed line.

the action quadratic in the fluctuation fields $\phi^{\prime}, \psi^{\prime}$ becomes

$$
\begin{aligned}
& S_{\text {quad }}^{\mathrm{II}}=-\frac{1}{2} \int \frac{d^{D} k}{(2 \pi)^{D}}\left(\phi^{\prime}(-k), \psi^{\prime}(-k)\right) \mathcal{M}^{\mathrm{II}}\left(k^{2}\right)\left(\begin{array}{c}
\phi^{\prime}(k) \\
\psi^{\prime}(k)
\end{array}\right), \\
& \mathcal{M}^{\mathrm{II}}\left(k^{2}\right)=\left(\begin{array}{cc}
k^{2}-1+\left(2 \phi^{\mathrm{II}}+2 \psi^{\mathrm{II}}\right) K^{-2 k^{2}} & \left(2 \phi^{\mathrm{II}}-\frac{13}{6}\right) K^{-2 k^{2}} \\
\left(2 \phi^{\mathrm{II}}-\frac{13}{6}\right) K^{-2 k^{2}} & k^{2}-4
\end{array}\right) .
\end{aligned}
$$

The determinant of the matrix $\mathcal{M}^{\mathrm{II}}\left(k^{2}\right)$ is plotted in Fig. 2. We see that $\operatorname{det} \mathcal{M}^{\mathrm{II}}$ vanishes only

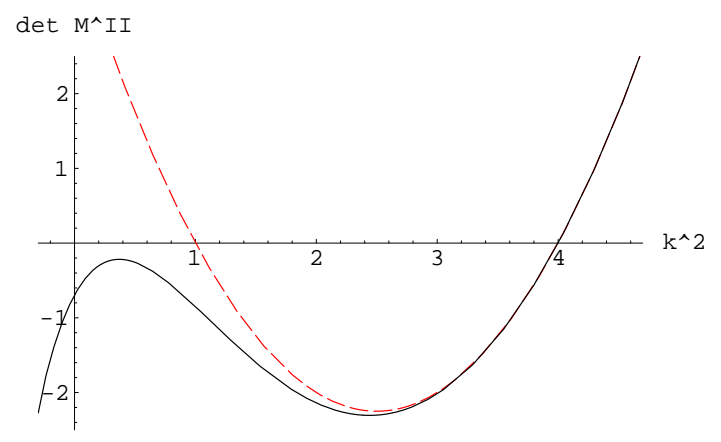

Figure 2: $\operatorname{det} \mathcal{M}^{\mathrm{II}}$ as a function of $k^{2}$ (solid line). There is no solution to $\operatorname{det} \mathcal{M}^{\mathrm{II}}=0$ around $k^{2}=1$.

once near $m_{c}^{2} \simeq-4.000$. This means that the perturbative spectrum around the solution (II) contains the closed string tachyon state, but not the open string tachyon. Since there is no physical open string state, we consider that the solution (II) corresponds to the background with no D-brane, i.e. the open string tachyon vacuum.

Now that we have obtained two vacuum configurations which can be interpreted as a Dbrane and the tachyon vacuum, we look for a time-dependent solution interpolating between 
them. The equations of motion following from the action (11) are

$$
\begin{aligned}
& \left(-\partial_{t}^{2}+1\right) K^{2 \partial_{t}^{2}} \widetilde{\phi}(t)-\widetilde{\phi}(t)^{2}+\frac{13}{6} \widetilde{\psi}(t)-2 \widetilde{\phi}(t) \widetilde{\psi}(t)=0, \\
& \left(-\partial_{t}^{2}+4\right) K^{2 \partial_{t}^{2}} \widetilde{\psi}(t)+\frac{13}{6} \widetilde{\phi}(t)-\widetilde{\phi}(t)^{2}=0
\end{aligned}
$$

where we have let $\phi$ and $\psi$ be functions only of the time variable $t$. The differential operators appearing in (10) can be rewritten as the convolution form [4, 12]

$$
\begin{aligned}
& \left(-\partial_{t}^{2}+\mu\right) K^{2 \partial_{t}^{2}} A(t)=\mathcal{C}^{(\mu)}[A](t) \\
& \quad \equiv \frac{1}{\sqrt{8 \pi \log K}} \int_{-\infty}^{\infty} d s\left(-\frac{(t-s)^{2}}{(4 \log K)^{2}}+\frac{1}{4 \log K}+\mu\right) e^{-\frac{(t-s)^{2}}{8 \log K}} A(s) .
\end{aligned}
$$

Then we can numerically solve the 'integral equations' (10) using the iterative procedure: Given a pair of the $n$-th functions $\phi_{n}(t)$ and $\psi_{n}(t)$, we can calculate the $(n+1)$-th functions as

$$
\begin{aligned}
\widetilde{\phi}_{n+1}(t) & =\frac{6}{13}\left(\widetilde{\phi}_{n}(t)^{2}-\mathcal{C}^{(4)}\left[\widetilde{\psi}_{n}\right](t)\right), \\
\widetilde{\psi}_{n+1}(t) & =\frac{6}{13}\left(-\mathcal{C}^{(1)}\left[\widetilde{\phi}_{n}\right](t)+\widetilde{\phi}_{n}(t)^{2}+2 \widetilde{\phi}_{n}(t) \widetilde{\psi}_{n}(t)\right) .
\end{aligned}
$$

If this iterative procedure converges, the set of functions $\widetilde{\phi}_{\infty}(t), \widetilde{\psi}_{\infty}(t)$ gives a solution to the equations of motion (10). Starting with the initial configurations

$$
\begin{aligned}
& \widetilde{\phi}_{0}(t)=\left\{\begin{array}{ll}
\phi^{\mathrm{I}}=0 & (t \leq 0) \\
\phi^{\mathrm{II}}=\frac{5}{6} & (t>0)
\end{array},\right. \\
& \widetilde{\psi}_{0}(t)=\left\{\begin{array}{ll}
\psi^{\mathrm{I}}=0 & (t \leq 0) \\
\psi^{\mathrm{II}}=-\frac{5}{18} & (t>0)
\end{array},\right.
\end{aligned}
$$

we have repeated the iterative procedure (12) 7000 times, and the resulting profiles for $\widetilde{\phi}_{7000}(t)$ and $\widetilde{\psi}_{7000}(t)$ are shown in Fig. 3. We have verified that $\widetilde{\phi}_{7000}(t)$ and $\widetilde{\phi}_{6000}(t)$ (and similarly for
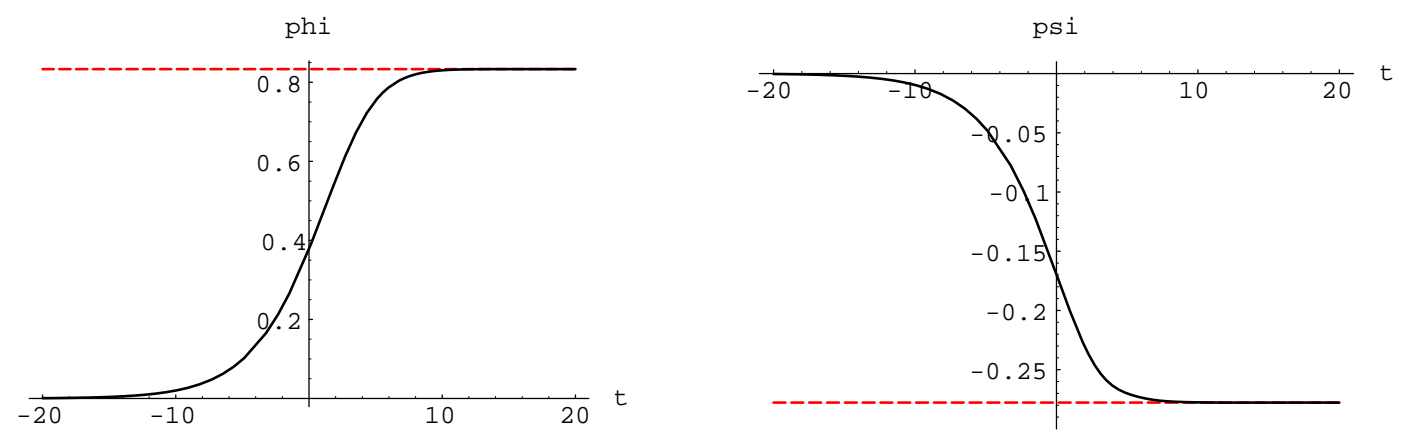

Figure 3: The solutions $\widetilde{\phi}(t)$ and $\widetilde{\psi}(t)$ after 7000 steps. The dashed lines show their expectation values $\left(\phi^{\mathrm{II}}, \psi^{\mathrm{II}}\right)$ at the tachyon vacuum. 
$\widetilde{\psi}_{7000}(t)$ and $\left.\widetilde{\psi}_{6000}(t)\right)$ completely agree with each other at least up to significant six digits for every value of $t$. We consider this as strong evidence that $\widetilde{\phi}_{n}(t)$ and $\widetilde{\psi}_{n}(t)$ really converge in the limit $n \rightarrow \infty$. Note that, since $\phi=\widetilde{\phi}$ holds within the range where $\phi$ is a constant, the tachyon fields $\phi, \psi$ indeed approach their vacuum values with vanishing velocity in this solution.

We will now check whether the energy is conserved in time for the solution obtained above. The energy $E(t)$ is calculated by the following formula [4]

$$
\begin{aligned}
& E(t)=E_{o}(t)+E_{c}(t)+E_{\text {int }}(t) \\
& E_{o}(t)=-\mathcal{L}_{o}+\sum_{l=1}^{\infty} \sum_{m=0}^{2 l-1}(-1)^{m}\left(\frac{\partial \mathcal{L}}{\partial \phi_{2 l}}\right)_{m} \phi_{2 l-m}, \\
& E_{c}(t)=-\mathcal{L}_{c}+\sum_{l=1}^{\infty} \sum_{m=0}^{2 l-1}(-1)^{m}\left(\frac{\partial \mathcal{L}}{\partial \psi_{2 l}}\right)_{m} \psi_{2 l-m}, \\
& E_{\text {int }}(t)=-\mathcal{L}_{\text {int }}
\end{aligned}
$$

where the subscripts denote the number of time-derivatives, $A_{n}(t) \equiv \frac{\partial^{n}}{\partial t^{n}} A(t)$. Although this formula contains infinite sums, we can obtain a good approximation to it by ignoring higher derivative terms, because they have negligibly small orders of magnitude as compared to $\phi$ and $\psi$ themselves, ${ }^{5}$ as shown in Fig. 4. Here we keep only up to the fourth derivatives. Note that it
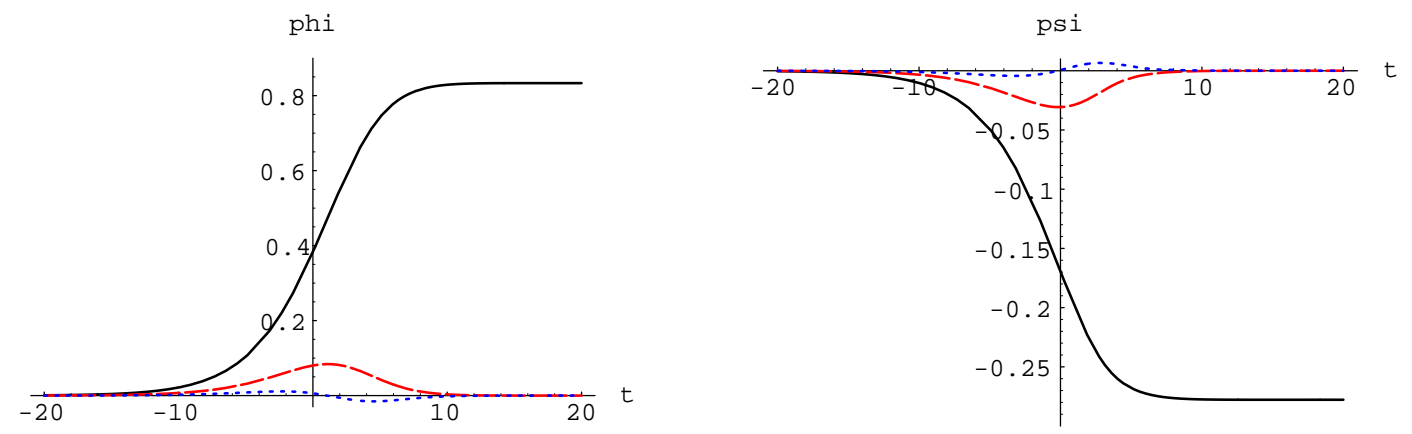

Figure 4: Plots of $\phi$ (left), $\psi$ (right) and their derivatives. The solid line shows $\phi(t)$ or $\psi(t)$, the dashed line their first derivatives, and the dotted line their second derivatives.

is meaningful to compare $\phi$ (or $\psi$ ) with its derivatives since the time variable $t$ has been made dimensionless by setting $\alpha^{\prime}=1$. The total energy $E(t)$ calculated this way is illustrated in Fig. [5A. If we compare it with Fig. $5 \mathrm{~B}$ where the constituents $E_{o}, E_{c}, E_{\text {int }}$ defined in (14) are shown separately, it is clear that highly non-trivial cancellations do occur among $E_{o}, E_{c}$ and $E_{\text {int }}$. We thus conclude that the total energy is constant $(=0)$ in time. However, as pointed out in [6], we do not know how to separate the total energy into the open and the closed string

\footnotetext{
${ }^{5}$ This also shows that the profile of $\widetilde{\phi}(\widetilde{\psi})$ is almost the same as that of $\phi(\psi)$.
} 

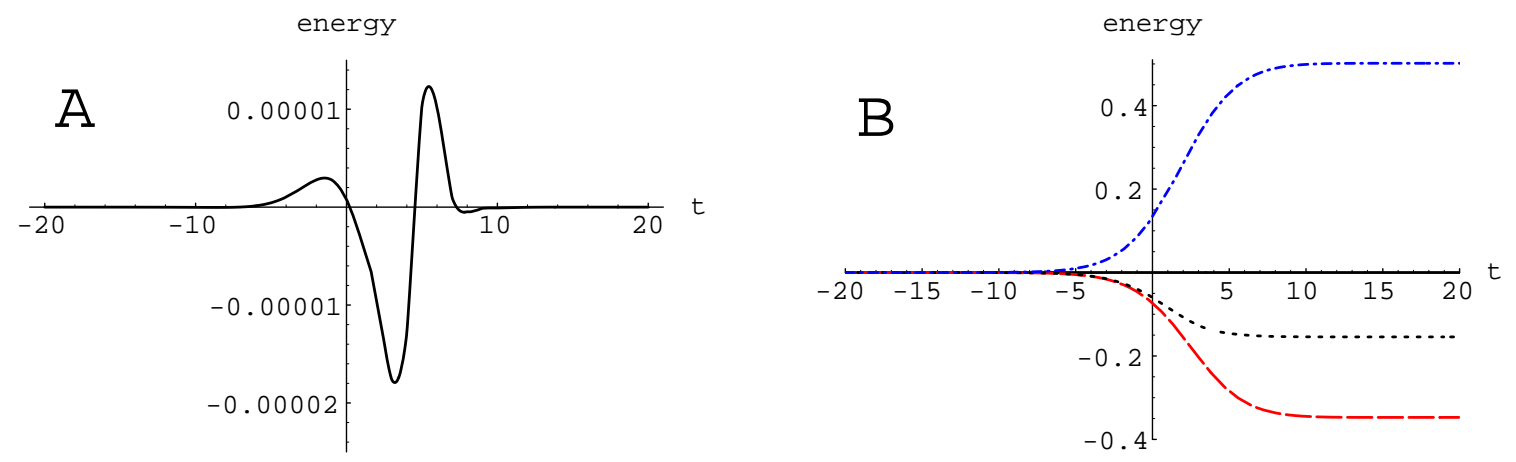

Figure 5: A: The total energy $E(t)$ calculated for our numerical solution. B: The energy decomposed into $E_{o}(t)$ (dashed line), $E_{c}(t)$ (dotted line) and $E_{\text {int }}(t)$ (dash-dotted line).

sectors. It would be interesting if we can see the flow of the energy from open to closed strings.

We have also investigated whether we can construct a time-dependent solution which interpolates between the solutions (I) and (III) using the iterative procedure, starting with

$$
\begin{aligned}
& \widetilde{\phi}_{0}(t)=\left\{\begin{array}{cc}
0 & (t \leq 0) \\
\phi^{\mathrm{III}}=\frac{5}{12} & (t>0)
\end{array},\right. \\
& \widetilde{\psi}_{0}(t)=\left\{\begin{array}{cc}
0 & (t \leq 0) \\
\psi^{\mathrm{III}}=-\frac{35}{192} & (t>0)
\end{array} .\right.
\end{aligned}
$$

From the result shown in Fig. [6] we see that the tachyon profiles tend to $\phi(t) \equiv \psi(t) \equiv 0$
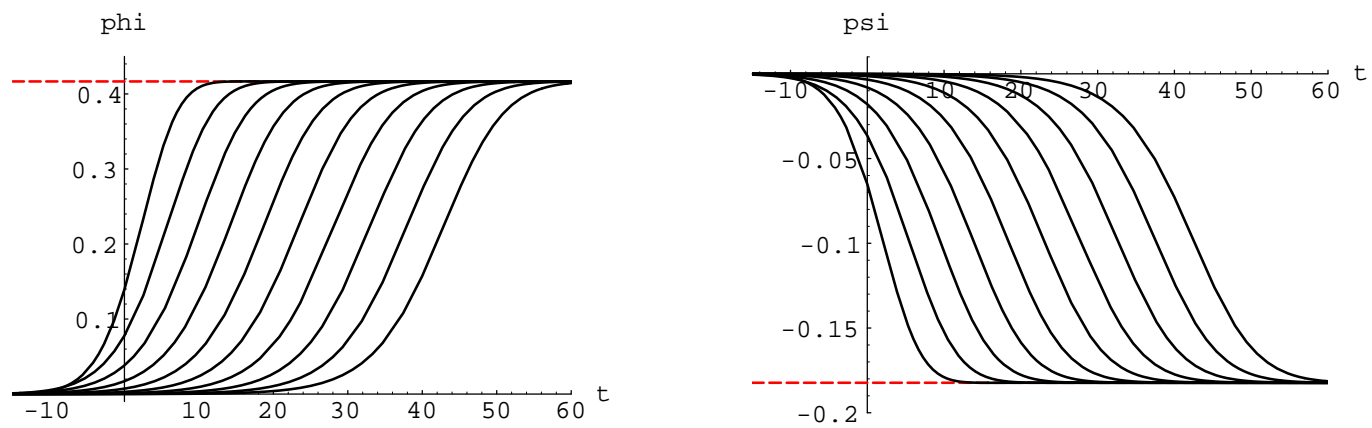

Figure 6: $\widetilde{\phi}_{n}(t)$ (left) and $\widetilde{\psi}_{n}(t)$ (right) for $n=10,20,30, \ldots, 100$ from left to right. The dashed lines show their expectation values $\left(\phi^{\mathrm{III}}, \psi^{\mathrm{III}}\right)$ at the solution (III).

as $n$ becomes large. Such a behavior is natural because there should be no energy-conserving solution connecting these two vacua in time. 


\section{Summary and Discussion}

In this paper we have argued that in the full open-closed string theory, the spatially homogeneous decay process of a space-filling unstable D-brane should be described by a time-dependent solution which interpolates between two static and energetically degenerate field configurations representing the unstable D-brane and the open string tachyon vacuum. In particular, to realize the situation in which the tachyon field converges to some finite value, it was essential to incorporate the bulk closed string modes because the open string sector must dissipate the energy which amounts to the D-brane tension through the couplings to closed strings. We have constructed and investigated a simple model describing the open and closed string tachyons, and found that (i) this model has two degenerate vacua which can be interpreted as the unstable D-brane and the tachyon vacuum, (ii) there exists a time-dependent solution which interpolates in time between them, and (iii) the total energy is conserved during the decay process described by this time-dependent solution. Hence it seems that some features expected of the rolling tachyon in open-closed string theory are indeed captured by our model.

There are many things to be studied further. We list some of them below.

- Among others, our analysis based on the toy model (11) is far from complete. In particular, there is no plausible justification for neglecting various higher-order interactions and for truncating the string fields to the tachyons. It would be interesting if we find that the time-dependent solution obtained in section 2 persists to exist after reinforcing our model by including higher-order interactions and other massless or massive fields.

- We have focused on the spatially homogeneous decay of a space-filling D-brane. In the case of the inhomogeneous decay or the decay of a lower-dimensional D-brane, the closed strings emitted from the decaying D-brane will be propagating outward in the transverse space. In this sense, the asymptotic state of the closed string field is not static. Nevertheless, we do expect that the open string field reaches the tachyon vacuum configuration sometime and ceases to evolve in time thereafter.

- We have identified the solution (II) with the open string tachyon vacuum by looking at the fluctuation spectrum around it. It is desirable to see that the energy stored in the open string field is lower at the solution (II) than at the solution (I). As mentioned in section 2. however, it is not known how to split the total energy into open and closed string sectors in our tachyon model. It may become possible if we can incorporate the metric degrees of freedom in our model, as in [8].

- It seems that the convolution form of the equations of motion is difficult to relate to the 'initial value problem' 1, 4. Our numerical solution corresponds to the rolling from the 
top of the potential with vanishing initial velocity, and has no parameter to be chosen freely. In fact, we have encountered a similar situation in seeking for static lump or kink solutions in (super)string field theory [13], where we did not treat it as a boundary value problem, and the width of the lump (kink) seemed to be determined automatically.

We hope that our investigation presented here sheds new light on the problem of the timedependent decay of unstable D-brane systems.

\section{Acknowledgments}

I am grateful to T. Eguchi, I. Kishimoto, N. Moeller, M. Schnabl and E. Watnabe for valuable discussions. I would also like to thank M. Fujii, Y. Matsuo, R. Nobuyama, K. Sakai, Y. Tachikawa and H. Takayanagi for useful conversations. The numerical computations in this work were performed using Mathematica. This work is supported by JSPS Research Fellowships for Young Scientists.

\section{References}

[1] A. Sen, "Rolling Tachyon," JHEP 0204 (2002) 048 hep-th/0203211; "Tachyon Matter," JHEP 0207 (2002) 065 hep-th/0203265.

[2] A. Sen, "Field Theory of Tachyon Matter," Mod. Phys. Lett. A17 (2002) 1797-1804 hep-th/0204143.

[3] S. Sugimoto and S. Terashima, JHEP 0207 (2002) 025 hep-th/0205085;

J.A. Minahan, JHEP 0207 (2002) 030 hep-th/0205098.

[4] N. Moeller and B. Zwiebach, "Dynamics with Infinitely Many Time Derivatives and Rolling Tachyons," JHEP 0210 (2002) 034 hep-th/0207107.

[5] M. Fujita and H. Hata, "Time Dependent Solution in Cubic String Field Theory," hep-th/0304163.

[6] N. Moeller and M. Schnabl, "Tachyon condensation in open-closed p-adic string theory," hep-th/0304213.

[7] T. Okuda and S. Sugimoto, Nucl. Phys. B647 (2002) 101-116 hep-th/0208196; A. Strominger, hep-th/0209090, B. Chen, M. Li and F.-L. Lin, JHEP 0211 (2002) 050 hep-th/0209222 ; F. Leblond and A.W. Peet, JHEP 0304 (2003) 048 hep-th/0303035;

N. Lambert, H. Liu and J. Maldacena, hep-th/0303139. 
[8] Y. Demasure and R.A. Janik, "Backreaction and the rolling tachyon - an effective action point of view," hep-th/0305191

[9] I. Ellwood, J. Shelton and W. Taylor, "Tadpoles and Closed String Backgrounds in Open String Field Theory," hep-th/0304259.

[10] B. Zwiebach, "Oriented Open-Closed String Theory Revisited," Annals Phys. 267 (1998) 193-248.

[11] T. Kugo and T, Takahashi, Prog. Theor. Phys. 99 (1998) 649-690 hep-th/9711100; T. Asakawa, T. Kugo and T. Takahashi, Prog. Theor. Phys. 100 (1998) 831-879 hep-th/9807066; Prog. Theor. Phys. 102 (1999) 427-466 hep-th/9905043; T. Kawano and T. Takahashi, Prog. Theor. Phys. 104 (2000) 1267-1287 hep-th/0005080.

[12] Y. Volovich, "Numerical Study of Nonlinear Equations with Infinite Number of Derivatives," math-ph/0301028.

[13] J.A. Harvey, and P. Kraus, JHEP 0004 (2000) 012 hep-th/0002117; R. de Mello Koch, A. Jevicki, M. Mihailescu and R. Tatar, Phys. Lett. B482 (2000) 249-254 hep-th/0003031; N. Moeller, A. Sen and B. Zwiebach, JHEP 0008 (2000) 039 hep-th/0005036; K. Ohmori, JHEP 0105 (2001) 035 hep-th/0104230. 